\title{
PREVALENCIA DE INFECCIÓN POR LOS VIRUS DE LA HEPATITIS B, D Y POR RETROVIRUS EN LA ETNIA MATSÉS (LORETO, PERÚ)
}

\author{
César Cabezas (1D) 1,2,a , Omar Trujillo (iD) ${ }^{2, \mathrm{c}}$, Johanna Balbuena (iD) ${ }^{2, \mathrm{~b}}$, Luis Marin (iD) ${ }^{2, \mathrm{~d}}$,

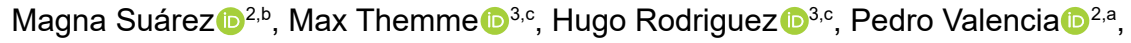 \\ Luis Crispin-Huamani (iD ${ }^{2,4, c}$ \\ ${ }^{1}$ Centro Nacional de Salud Pública, Instituto Nacional de Salud, Lima, Perú. \\ ${ }^{2}$ Facultad de Medicina Humana, Universidad Nacional Mayor de San Marcos, Lima, Perú. \\ ${ }^{3}$ Dirección Regional de Salud de Loreto, Perú. \\ ${ }^{4}$ Asociación para el Desarrollo de la Investigación Estudiantil en Ciencias de la Salud, Universidad Nacional Mayor de \\ San Marcos, Lima, Perú. \\ ${ }^{a}$ Médico infectólogo tropicalista; ${ }^{\mathrm{b}}$ bióloga; ${ }^{\mathrm{c}}$ médico cirujano; ${ }^{\mathrm{d}}$ tecnólogo médico
}

\section{RESUMEN}

Para determinar la prevalencia de infección por los virus de la hepatitis B y D (VHB y VHD, respectivamente), VIH y HTLV-1/2 en la etnia matsés, después de la inmunización contra el VHB se realizó un estudio transversal y poblacional, utilizando pruebas de ELISA y qPCR en 963 pobladores. Las prevalencias de $\mathrm{HBsAg}$, anti-HBc y anti-HBs fueron 3,3\%, 36,0\% y 58,7\%, respectivamente. En el 3,1\% de la población la carga viral fue mayor a $2000 \mathrm{UI} / \mathrm{mL}$. En menores de 10 años, la prevalencia de $\mathrm{HBs} A g$ y anti-HBc fue $0,0 \%$ y $2,6 \%$, respectivamente, mientras que en el $94,4 \%$ se encontraron anticuerpos protectores. La prevalencia de infección por el VIH y el HTLV-1/2 fue 1,5\% y 0,6\%, respectivamente. Se concluye que existen tasas bajas de infección por el VHB y el VHD en la población infantil de la etnia matsés. Asimismo, se confirma la presencia de infección por el VIH y el HTLV-1/2.

Palabras clave: Hepatitis B; Inmunización; VIH; HTLV; Comunidades Indígenas (fuente: DeCS BIREME).

\section{PREVALENCE OF HBV, HDV AND RETROVIRUS INFECTION IN THE MATSÉS ETHNIC GROUP IN LORETO, PERU}

\begin{abstract}
Observational, cross-sectional, populational study to determine the prevalence of infection by hepatitis B virus (HBV), hepatitis D virus (HDV), human immunodeficiency virus (HIV) and human T-lymphotropic virus type 1 and 2 (HTLV-1/2) in the Matsés ethnic group, after immunization against HBV. ELI$\mathrm{SA}$ and qPCR tests were used in 963 residents. The prevalence of $\mathrm{HBsAg}$, Anti-HBc and Anti-HBs was $3.32 \%, 36.03 \%$ and $58.67 \%$ respectively. In $3.1 \%$ of the population the viral load was greater than 2000 $\mathrm{IU} / \mathrm{mL}$. In children under 10 years, the prevalence of $\mathrm{HBsAg}$ and anti-HBc was $0.0 \%$ and $2.6 \%$, respectively, while protective antibodies were found in $94.4 \%$. The prevalence of HIV and HTLV- $1 / 2$ infection was $1.5 \%$ and $0.6 \%$, respectively. It is therefore concluded that there are low rates of HBV and HDV infection in the Matsés child population. Likewise, the presence of HIV and HTLV-1/2 infection is confirmed.
\end{abstract}

Keywords: Hepatitis B; Immunization; HIV; HTLV; Indigenous Population (source: MeSH NLM).

Citar como: Cabezas C, Trujillo O, Balbuena J, Marin L, Suárez M, Themme M, et al. Prevalencia de infección por los virus de la hepatitis $\mathrm{B}, \mathrm{D}$ y por retrovirus en la etnia Matsés (Loreto, Peru). Rev Peru Med Exp Salud Publica. 2020;37(2):25964. doi: https://doi.org/10.17843/ rpmesp.2020.372.4696

Correspondencia: César Cabezas Sánchez; ccabezas@ins.gob.pe

Recibido: $24 / 07 / 2019$

Aprobado: $06 / 05 / 2020$

En línea: 01/06/2020

\section{INTRODUCCIÓN}

En el mundo es conocida la endemicidad de la infección por el virus de las hepatitis B y D (VHB y VHD) ${ }^{(1)}$, principalmente en África ${ }^{(2)}$. Si bien los países de Sudamérica son considerados de baja endemicidad, existen pueblos indígenas de la Amazonía peruana ${ }^{(6)}$ y comunidades indígenas en el Brasil, Colombia y Venezuela que presentan una alta prevalencia de esta infección ${ }^{(3-5)}$.

Las coinfecciones del VHB con los retrovirus como el virus de inmunodeficiencia humana (VIH) y el virus linfotrópico de células T humanas (HTLV, por sus siglas en inglés) tipo 1 y 2 hacen más compleja la prevención y el control de estas infecciones ${ }^{(7)}$, sobre todo en zonas rurales con poco acceso a los servicios de salud, como en las comunidades indígenas de la Amazonía ${ }^{(8)}$. En estas comunidades también se han descrito prevalencias de 5,9\% y 3,8\% 
de infecciones por HTLV-1 y HTLV-2, respectivamente ${ }^{(9,10)}$. Pese a que estas infecciones pueden influir en la sobrevivencia de dichas comunidades, ya se cuenta con programa de vacunación de VHB que ha demostrado una disminución de la enfermedad, principalmente en la población infantil ${ }^{(11)}$.

En este contexto es necesario conocer la situación actual de las infecciones por VHB y VHD (prevenibles con la vacunación), y de las infecciones por VIH y HTLV-1/2 en las poblaciones indígenas de la Amazonía peruana. Estudios similares se han realizado en otros países de la región amazónica como Colombia y Venezuela ${ }^{(12,13)}$.

Por lo tanto, el objetivo del estudio fue determinar la prevalencia de infección por VHB, VHD, VIH y HTLV-1/2 en la etnia matsés, población indígena que reside en la Amazonía peruana.

\section{EL ESTUDIO}

\section{Población y tamaño muestral}

Se realizó un estudio transversal y poblacional en las 14 comunidades indígenas de la etnia matsés, ubicadas en las cuencas del Yavari, Yaquerana, Gálvez y la quebrada Chobayacu, del distrito de Yaquerana, provincia de Requena, región de Loreto (Figura 1). Según el censo del 2007 del Instituto Nacional de Estadística e Informática (INEI), la población estimada fue 1724 personas. Estas comunidades fueron visitadas durante el 2012.

Se incluyeron en el estudio a todos los pobladores que se encontraban en sus viviendas durante las visitas, no se rea-

\section{MENSAJES CLAVE}

Motivación para realizar el estudio: Los pueblos indígenas de la Amazonía peruana podrían seguir teniendo altas tasas de infección por los virus de la hepatitis $\mathrm{B}$ y D, pese a la inmunización.

Principales hallazgos: La prevalencia de $\mathrm{HBsAg}$, anti-HBc total y anti-HBs fue $3,3 \%, 36,0 \%$ y $58,7 \%$, respectivamente, siendo mayor el HBsAg y anti-HBc en la población adulta. En menores de 10 años, la prevalencia de HBsAg y anti-HBc fue $0,0 \%$ y $2,6 \%$ respectivamente, mientras que en el $94,4 \%$ se encontraron anticuerpos protectores.

Implicancias: En la población indígena es necesario realizar intervenciones culturalmente adecuadas para controlar y diagnosticar de manera oportuna las enfermedades infectocontagiosas.

lizó un cálculo previo de una muestra, ya que la intención era incluir al total de la población indígena, considerando ambos sexos y a todos los grupos etarios. El personal de salud aplicó de forma confidencial una encuesta epidemiológica estructurada en la vivienda de cada poblador, la cual recogió información de los antecedentes personales (sexo, edad, fecha de nacimiento, procedencia) y del estado de inmunización a través del carné de vacunación. Para asegurar la comprensión de las preguntas se contó con la presencia de traductores.

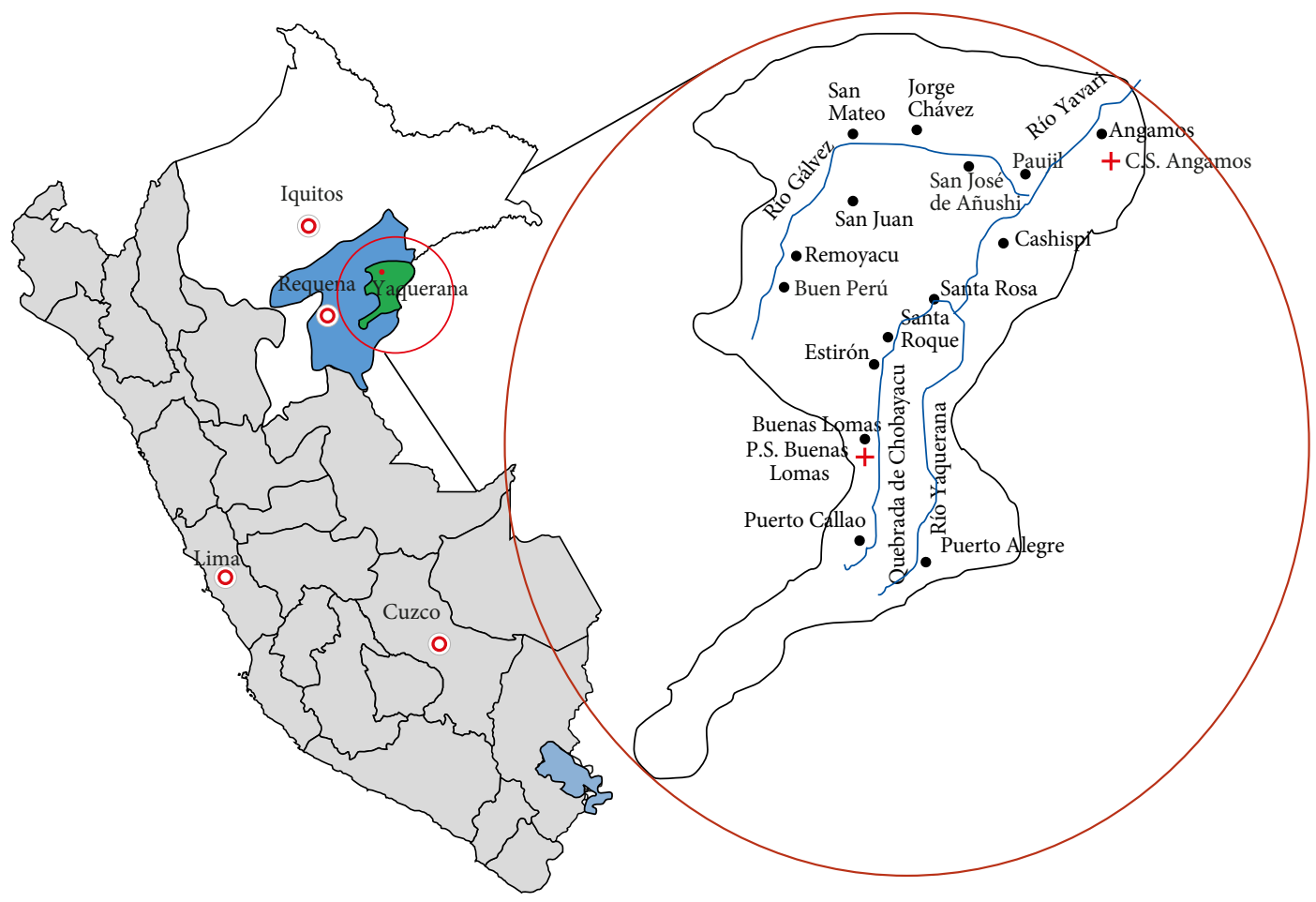

Figura 1. Ubicación geográfica de la etnia matsés en la Amazonía peruana 
Se consideró infección crónica por VHB cuando el HBsAg (antígeno de superficie de la hepatitis B, por sus siglas en inglés) $\mathrm{y}$ anti-HBc (anticuerpo contra el antígeno core de la hepatitis B) eran positivos y el anti-HBc-IgM era negativo. Infección pasada, cuando el anti-HBc y el anti-HBs (anticuerpo contra el HBsAg) eran positivos y el HBsAg era negativo. Infección aguda, cuando el HBsAg y el antiHBc-IgM eran positivos. Susceptibles, cuando el HBsAg, anti-HBc y anti-HBs eran negativos. Si solo el anti-HBs era positivo, se consideró estado de protección. Los niveles de anti-HBs $\geq 10 \mathrm{mUI} / \mathrm{ml} \mathrm{se}$ consideraron protectores.

\section{Análisis de laboratorio}

Se obtuvieron muestras de $10 \mathrm{~mL}$ de sangre de los pobladores mayores de 6 años, y de $5 \mathrm{~mL}$ de sangre de los menores de 6 años. Estas muestras fueron procesadas en el Laboratorio de Referencia Nacional de Hepatitis y de VIH del Instituto Nacional de Salud (INS). Se utilizó la técnica de ELISA para determinar los marcadores serológicos de VHB, VHD, VIH y HTLV-1/2.

Para determinar la infección por VHB se evaluaron los marcadores HBsAg, anti-HBc y anti-HBs (BioElisa). Las muestras con resultado positivo para el HBsAg fueron evaluadas para detectar el HBeAg y anti-HBc IgM (Wantai), las muestras anti- $\mathrm{HBc}$ positivas fueron evaluadas para detecar anti-VHD IgM y anti-HDV-IgG (Wantai). Todas las pruebas se realizaron en el lavador y lector de ELISA (Robonik). Se determinó la carga viral de los participantes con infección crónica por el VHB mediante el método de reacción en cadena de la polimerasa en tiempo real cuantitativa (qPCR), utilizando los kits $\mathrm{COBAS}^{\oplus}$ TaqMan $^{\circledR}$ HBV Test-High Pure System (Roche) en el termociclador Cobas TaqMan 48, cuyo límite inferior de detección fue $29 \mathrm{UI} / \mathrm{mL}$. Para determinar la infección por VIH y HTLV-1/2 se utilizaron pruebas ELISA (BioElisa) y LIA (Inmunogenetic).

\section{Análisis estadístico}

El análisis estadístico se realizó con el programa Stata 14.0, (College Station, Texas) para Windows. Se calcularon las prevalencias del VHB, VHD, VIH y HTLV-1/2 en población indígena según edad, sexo y procedencia, acompañadas de sus intervalos de confianza al 95\% (IC 95\%).

\section{Consideraciones éticas}

Se obtuvo el consentimiento informado colectivo de parte de las organizaciones indígenas representativas (regionales) y de los entes de máxima jerarquía (Apus) y el consentimiento informado individual de los pobladores matsés, en los niños mayores de 6 años se obtuvo un asentimiento informado. El protocolo del estudio fue aprobado por el Comité de Ética e Investigación del INS. Al finalizar los análisis de laboratorio los resultados fueron entregados a la comunidad y a las personas participantes del estudio.

\section{HALLAZGOS}

Se obtuvieron 963 muestras sanguíneas. La media de la edad de la población fue de 23 años (mín., 1 - máx., 80) y el 52,1\% fueron mujeres. Los menores de 5 años representaron el 11,7\% (102/871) y los menores de 10 años, el 26,75\% (233/871).

La prevalencia del HBsAg, anti-HBc y anti-HBs fue 3,3\% $(\mathrm{n}=32), 36,0 \%(\mathrm{n}=347)$ y $58,7 \%(\mathrm{n}=565)$, respectivamente, siendo mayores las prevalencias del HBsAg y anti-HBc en los adultos de 30 a 59 años. En los menores de 10 años, las prevalencias del HBsAg y anti-HBc fueron $0,0 \%$ y $2,6 \%$ (6/233), respectivamente. El 94,4\% (220/233) tuvieron anticuerpos protectores (anti-HBs) sin anticuerpos que indicaran infección pasada (anti-HBc) (Tabla 1).

El 3,0\% (7/233) de los participantes no presentó anticuerpos protectores ni marcadores serológicos de infección pasada por hepatitis B; el 0,4\% (1/233), coinfección VHBVHD; y el 3,3\% (n=32), infección crónica por el VHB. Todos los participantes resultaron negativos para HBeAg. Respecto a la carga viral del $\mathrm{VHB}$, solo $1 / 32(3,1 \%)$ fue mayor a $2000 \mathrm{UI} / \mathrm{mL}$ en los portadores crónicos. El 3,2\% (31/963) tuvo coinfección VHB-VHD.

La prevalencia de infección por el VIH y HTLV-1/2 fue $1,5 \%(12 / 806)$ y $0,6 \%(5 / 806)$, respectivamente. La prevalencia de HTLV-1/2 en los varones fue $1,1 \%$ (4/368) y la prevalencia de VIH en niños menores de 10 años fue $2,7 \%$ $(6 / 220)$. Los infectados con VIH fueron mayores de 2 años mientras que los infectados con HTLV-1/2 fueron mayores de 21 años. La prevalencia por sexo y edad, así como los resultados de marcadores de infección por VIH y HTLV-1/2 en población total estudiada se muestran en la Tabla 2.

En cuanto a las coinfecciones, el 0,5\% (4/806) tuvo coinfección VHB-VIH, y el 0,4\% (3/808), coinfección VHBHTLV-1/2. Mientras que para quienes tuvieron infección por VHB las coinfecciones con VHD, VIH y HTLV-1/2 fueron de $8,9 \%(31 / 347), 1,4 \%(4 / 285)$ y $1,0 \%(3 / 286)$, respectivamente.

El antecedente de vacunación contra el VHB, referida por las madres de niños menores de 5 años con tarjeta de vacunación, fue del 33,0\% (9/27).

\section{DISCUSIÓN}

Las infecciones por los virus de hepatitis, así como sus consecuencias, siempre han representado una preocupación en las comunidades amazónicas como la matsés, ubicada entre Brasil y Perú. Los hallazgos de este estudio son alentadores si se comparan las prevalencias obtenidas en la población infantil con otros estudios realizados en la zona ${ }^{(14)}$ y en las comunidades indígenas de la Amazonía peruana ${ }^{(6)}$. La prevalencia de infección de los matsés es similar a la descrita en otras comunidades amazónicas de Colombia y Venezuela, 
Tabla 1. Prevalencia de los marcadores para virus de hepatitis B y D en la etnia matsés, distrito de Yaquerana, provincia de Requena, Loreto, Perú, 2012

\begin{tabular}{|c|c|c|c|c|c|c|c|c|c|c|}
\hline \multirow{3}{*}{ Grupos } & \multirow{3}{*}{$\mathrm{N}(\%)$} & \multicolumn{2}{|c|}{ HBsAg } & \multicolumn{2}{|c|}{ Anti-HBc total } & \multicolumn{2}{|c|}{$\begin{array}{l}\text { Anti-HBs en protegidos } \\
\text { contra VHB }^{\text {a }}\end{array}$} & \multirow{3}{*}{$\mathrm{N}(\%)$} & \multicolumn{2}{|c|}{ VHD } \\
\hline & & Positivos & Prevalencia & Positivos & Prevalencia & Positivos & Prevalencia & & Positivo & Prevalencia \\
\hline & & n (\%) & IC 95\% & n (\%) & IC 95\% & n (\%) & IC 95\% & & n (\%) & IC 95\% \\
\hline $\begin{array}{l}\text { Todos los } \\
\text { participantes }\end{array}$ & $963(100)$ & $32(3,3)$ & $(2,3-4,6)$ & $347(36,0)$ & $(33,0-39,2)$ & $565(58,7)$ & $(55,5-61,8)$ & $335(100)$ & $31(9,2)$ & $(6,4-12,9)$ \\
\hline \multicolumn{11}{|l|}{ Sexo } \\
\hline Mujeres & $502(52,1)$ & $15(3,0)$ & $(1,7-4,9)$ & $177(35,3)$ & $(31,1-39,7)$ & $301(59,9)$ & $(55,5-64,3)$ & $170(50,7)$ & $12(7,1)$ & $(3,7-12,0)$ \\
\hline Varones & $461(47,9)$ & $17(3,7)$ & $(2,16-5,84)$ & $170(36,9)$ & $(32,4-41,4)$ & $264(57,3)$ & $(52,6-61,8)$ & $165(49,2)$ & $19(11,5)$ & $(7,1-17,4)$ \\
\hline \multicolumn{11}{|l|}{$\begin{array}{l}\text { Grupo etario } \\
\text { (años) }^{\mathrm{b}}\end{array}$} \\
\hline De 0 a 10 & $259(29,1)$ & $0(0,0)$ & $(0,0-0,0)$ & $6(2,3)$ & $(0,8-4,9)$ & $245(94,6)$ & $(91,1-97,0)$ & $5(1,6)$ & $1(0,2)$ & $(0,5-71,6)$ \\
\hline De 11 a 18 & $176(19,8)$ & $3(1,7)$ & $(0,3-4,9)$ & $14(7,9)$ & $(4,4-12,9)$ & $158(89,8)$ & $(84,3-93,8)$ & $14(4,6)$ & $1(7,1)$ & $(0,2-33,9)$ \\
\hline De 19 a 29 & $175(19,7)$ & $10(5,7)$ & $(2,8-10,3)$ & $85(48,6)$ & $(40,9-56,2)$ & $73(41,7)$ & $(34,3-49,4)$ & $83(27,2)$ & $14(16,9)$ & $(9,5-26,7)$ \\
\hline De 30 a 59 & $251(28,2)$ & $17(6,8)$ & $(3,9-10,6)$ & $184(73,3)$ & $(67,4-78,7)$ & $55(21,9)$ & $(16,9-27,5)$ & $179(58,7)$ & $14(7,8)$ & $(4,3-12,8)$ \\
\hline$\geq 60$ & $29(3,3)$ & $2(6,9)$ & $(0,8-22,8)$ & $25(86,2)$ & $(68,3-96,1)$ & $3(10,3)$ & $(2,2-27,3)$ & $24(7,9)$ & $0(0,0)$ & $(0,0-0,0)$ \\
\hline \multicolumn{11}{|l|}{ Menores de 5 años } \\
\hline Sí & $102(11,7)$ & $0(0,0)$ & $(0,0-0,0)$ & $3(2,8)$ & $(0,6-7,9)$ & $98(91,6)$ & $(84,6-96,1)$ & $2(0,7)$ & $1(50,0)$ & $(1,3-98,7)$ \\
\hline No (5 a más) & $769(88,3)$ & $32(4,2)$ & $(2,9-5,8)$ & $311(40,4)$ & $(36,9-44,0)$ & $432(56,1)$ & $(52,6-59,7)$ & $303(99,3)$ & $29(9,6)$ & $(6,5-13,5)$ \\
\hline \multicolumn{11}{|l|}{ Cuenca $^{c}$} \\
\hline Yavarí & $481(50,1)$ & $14(2,9)$ & $(1,6-4,8)$ & $208(43,2)$ & $(38,8-47,8)$ & $252(52,3)$ & $(47,8-56,9)$ & $29(8,7)$ & $3(10,3)$ & $(2,2-27,4)$ \\
\hline Gálvez & $68(7,1)$ & $4(5,9)$ & $(1,6-14,4)$ & $29(42,6)$ & $(30,7-55,2)$ & $33(48,5)$ & $(36,2-60,9)$ & $52(15,6)$ & $4(7,7)$ & $(2,1-18,5)$ \\
\hline Yaquerana & $165(17,2)$ & $7(4,2)$ & $(1,7-8,5)$ & $53(32,1)$ & $(25,1-39,8)$ & $107(64,8)$ & $(57,0-72,1)$ & $53(15,9)$ & $6(11,3)$ & $(4,3-23,0)$ \\
\hline $\begin{array}{l}\text { Quebrada } \\
\text { Chobayacu }\end{array}$ & $248(25,8)$ & $7(2,8)$ & $(1,1-5,7)$ & $56(22,6)$ & $(17,5-28,3)$ & $173(69,8)$ & $(63,6-75,4)$ & $200(59,9)$ & $18(9,0)$ & $(5,4-13,8)$ \\
\hline
\end{tabular}

${ }^{a}$ Corresponde a 565 participantes (HBsAg=negativo, anti-HBc=negativo, anti-HBs=positivo)

${ }^{\mathrm{b}}$ El reporte del grupo etario fue obtenido de 871 participantes

${ }^{c}$ El reporte de las cuencas fue obtenido de 962 participantes

donde también se observa una significativa reducción de las tasas de portadores y de infección por $\mathrm{VHB}{ }^{(12,13)}$.

El presente estudio muestra que el 91,6 \% de los niños menores de 5 años tienen anti-HBs, es decir, se encuentran en un estado de protección, lo cual es compatible con la ausencia de portadores y con la muy baja tasa de infección por $\mathrm{VHB}$ en relación con la población total. Este hallazgo coincide con lo descrito en una región amazónica de Colombia ${ }^{(15)}$, donde el $93 \%$ de los niños se encontraban vacunados, lo que refleja la baja tasa de infección $(3,6 \%)$ y el poco porcentaje de portadores crónicos de HBsAg $(0,5 \%)$, aunque en este estudio no se determinaron anticuerpos anti-HBs.

La infección por el VHD en los casos positivos para HBsAg fue 9,3\%, lo que estaría en relación con la disminución de portadores del VHB en respuesta a la inmunización, resultado similar se observó en un estudio en Túnez ${ }^{(16)}$.

La prevalencia del VIH en esta etnia fue $1,5 \%$, sin embargo, son notorias las prevalencias del $2,9 \%$ y $2,3 \%$ en menores de 10 años y en menores de 5 años, respectivamente. Estos hallazgos son superiores al 0,7\% encontrado por Bartlett et al. ${ }^{(14)}$ en Yurimaguas y al $0.16 \%$ encontrado por Ormaeche et al. ${ }^{(17)}$. No obstante, en la etnia de los chayahuitas de la Amazonía peruana se ha encontrado una prevalencia del $7,5 \%{ }^{(18)}$.
En este estudio se encontró una coinfección VHB-VIH de $0,5 \%$ y una coinfección VHB-HTLV de $0,4 \%$, cifras inferiores al 7,0\% de coinfección VHB-VIH observado por Mathews et al. en comunidades africanas ${ }^{(19)}$.

Respecto a la infección por HTLV-1/2 en la etnia matsés, se encontró una prevalencia del $0,6 \%$, inferior a la encontrada en las comunidades shipibo-konibo de la Amazonía peruana, donde Blas et al. ${ }^{(10)}$ encontraron una prevalencia del 9,7\%. Así, nuestros hallazgos son más parecidos al 1,5\% de prevalencia observado por Medeot et al. en comunidades de la Amazonía peruana ${ }^{(9)}$ y al $0,8 \%$ encontrado por Bittencourt et al. en Brasil ${ }^{(20)}$.

El antecedente de vacunación contra el VHB en menores de 5 años fue 33,0\%, pero si tenemos en cuenta los resultados de la presencia de anticuerpos protectores anti-HBs y la baja prevalencia de marcadores de infección, el dato encontrado no es consistente con los resultados serológicos, que representan la mejor evidencia, tanto del estado de protección como de infección. Esto fue descrito por Fiestas et al., quienes mostraron que la información sobre la cobertura de vacunación obtenida de la Encuesta Demográfica y de Salud Familiar no concuerda con los niveles de protección contra la sarampión, la rubeola y el HVB ${ }^{(21)}$. 
Tabla 2. Prevalencia del VIH y HTLV-1/2 en la etnia matsés, distrito de Yaquerana, provincia de Requena, Loreto, Perú, 2012

\begin{tabular}{|c|c|c|c|c|c|c|}
\hline \multirow{3}{*}{ Grupos } & \multirow{3}{*}{$\mathbf{N}(\%)$} & \multicolumn{2}{|c|}{ VIH $^{b}$} & \multirow[b]{2}{*}{$\mathbf{N}(\%)$} & \multicolumn{2}{|c|}{ HTLV- $1 / 2^{c}$} \\
\hline & & Positivo & Prevalencia & & Positivo & Prevalencia \\
\hline & & n (\%) & IC 95\% & & n (\%) & IC 95\% \\
\hline Todos los participantes & $806(100)$ & $12(1,5)$ & $(0,8-2,6)$ & $807(100)$ & $5(0,6)$ & $(0,2-1,4)$ \\
\hline \multicolumn{7}{|l|}{ Sexo } \\
\hline Mujeres & $439(54,5)$ & $7(1,6)$ & $(0,6-3,3)$ & $439(54,5)$ & $1(0,2)$ & $(0,0-1,3)$ \\
\hline Varones & $367(45,5)$ & $5(1,4)$ & $(0,4-3,1)$ & $368(45,5)$ & $4(1,1)$ & $(0,3-2,8)$ \\
\hline \multicolumn{7}{|l|}{ Grupo etario (años) a } \\
\hline De 0 a 10 & $220(29,1)$ & $6(2,7)$ & $(1,0-5,8)$ & $221(27,8)$ & $0(0,0)$ & $(0,0-0,0)$ \\
\hline De 11 a 18 & $141(18,6)$ & $0(0,0)$ & $(0,0-0,0)$ & $141(18,1)$ & $0(0,0)$ & $(0,0-0,0)$ \\
\hline De 19 a 29 & $156(20,6)$ & $2(1,3)$ & $(0,2-4,5)$ & $156(19,3)$ & $2(1,3)$ & $(0,2-4,5)$ \\
\hline De 30 a 59 & $216(28,6)$ & $4(1,8)$ & $(0,5-4,7)$ & $217(26,9)$ & $3(1,4)$ & $(0,3-4,0)$ \\
\hline$\geq 60$ & $23(3,0)$ & $0(0,0)$ & $(0,0-0,0)$ & $23(2,8)$ & $0(0,0)$ & $(0,0-0,0)$ \\
\hline \multicolumn{7}{|l|}{ Cuenca $^{b}$} \\
\hline Yavarí & $352(43,7)$ & $10(2,8)$ & $(1,4-5,2)$ & $354(43,9)$ & $4(1,1)$ & $(0,3-2,9)$ \\
\hline Gálvez & $68(8,4)$ & 0,0 & $(0,0-0,0)$ & $68(8,4)$ & $0(0,0)$ & $(0,0-0,0)$ \\
\hline Yaquerana & $138(17,1)$ & $1(0,7)$ & $(0,0-4,0)$ & $138(17,1)$ & $1(0,7)$ & $(0,0-3,9)$ \\
\hline Quebrada Chobayacu & $247(30,7)$ & $1(0,4)$ & $(0,0-2,2)$ & $247(30,6)$ & $0(0,0)$ & $(0,0-0,0)$ \\
\hline
\end{tabular}

${ }^{a}$ El reporte del grupo etario fue obtenida de 756 participantes voluntarios

${ }^{\mathrm{b}} \mathrm{El}$ reporte de las cuencas fue obtenido de 805 participantes voluntarios

${ }^{c}$ El reporte de las cuencas fue obtenido de 807 participantes voluntarios

Se deben mencionar algunas limitaciones del estudio: primero, la población representó el $57 \%$ de la población estimada, debido a que en el momento de la intervención muchos pobladores no se encontraban en sus viviendas. Segundo, no se realizó ningún estudio antes del programa de inmunización, lo que hubiera servido para evaluar objetivamente una variación más exacta. Tercero, la ausencia de información precisa sobre las coberturas de vacunación limita el estimado final, aunque los marcadores serológicos de infección por VHB brindan información sobre el estado de infección de la población estudiada.

En conclusión, en la etnia matsés la prevalencia de la infección por VHB y VHD fue 3,3\% y 9,3\%, respectivamente. Además, el $94,6 \%$ de niños menores de 10 años se encuentran en estado de protección por tener anti-HBs, esto último puede estar relacionado con la inmunización previa realizada. Se confirma la presencia de infección por el VIH y HTLV-1/2, lo que constituye un riesgo para la misma etnia y para otros grupos de la frontera peruano-brasilera, siendo necesario adoptar intervenciones culturalmente adecuadas para elaborar un diagnóstico oportuno, brindar un tratamiento antirretroviral, hacer un seguimiento de los casos y tomar las medidas preventivas. Amerita realizar estudios cualitativos sobre las conductas sexuales y de riesgo para que las intervenciones que se desarrollen tengan mejores probabilidades de éxito, ademas de continuar con los programas de inmunización para el VHB.

Contribuciones de los autores: CC concibió y diseñó el estudio. CC, OT, JB, LM, MS, MT, HR y PV contribuyeron con la recolección de la información y la redacción del artículo. CC y LCH se encargaron de la revisión crítica del artículo. OT, JB y LM contribuyeron con el análisis e interpretación de datos y la asesoría estadística. MS, MT, HR y PV contribuyeron con el aporte de pacientes o material de estudio, obtención de financiamiento y asesoría técnica o administrativa. CC, OT, JB, LM, MS, MT, HR, PV y LCH aprobaron la versión final del artículo.

Financiamiento: Instituto Nacional de Salud de Perú.

Conflictos de interés: Los autores no tienen ningún conflicto de interés que declarar.

\section{REFERENCIAS BIBLIOGRÁFICAS}

1. World Health Organization. Global hepatitis report, 2017 [Internet]. Geneva: Department of HIV and Global Hepatitis Programme of the World Health Organization, WHO; 2017 (citado el 06 de noviembre de 2019). Disponible en: http://apps.who.int/iris/bitstream/10665/255016 /1/9789241565455-eng.pdf?ua $=1$.

2. Musa BM, Bussell S, Borodo MM, Samaila AA, Femi OL. Prevalence of hepatitis B virus infection in Nigeria, 2000-2013: a systematic review and meta-analysis. Niger J Clin Pract. 2015;18(2):163-72. doi: 10.4103/1119-3077.151035.
3. Dutra F. Distribution of hepatitis B infection in Brazil: the epidemiological situation at the beginning of the 21st century. Revista da Sociedade Brasileira de Medicina Tropical. 2016;49(1):11-23. doi: 10.1590/00378682-0176-2015.

4. di Filippo Villa D, Cortes-Mancera F, Payares E, Montes N, de la Hoz F, Arbelaez MP, et al. Hepatitis D virus and hepatitis B virus infection in Amerindian communities of the Amazonas state, Colombia. Virol J. 2015;12:172. doi: 10.1186/s12985-015-0402-5. 
5. Graham S, Guy RJ, Cowie B, Wand H, Donovan B, Akre S, et al. Chronic hepatitis B prevalence among Aboriginal and Torres Strait Islander Australians since universal vaccination: a systematic review and meta-analysis. BMC Infect Dis. 2013;13:403. doi: 10.1186/1471-2334-13-403.

6. Cabezas C, Suarez M, Romero G, Carrillo C, García M, Reátegui J, et al. Hiperendemicidad de hepatitis viral B y Delta en pueblos indígenas de la Amazonía Peruana. Rev Peru Med Exp Salud Publica. 2006;23(2):114-22.

7. Alves da Costa C, Okamura L. Molecular epidemiology of hepatitis B virus among the indigenous population of the Curuçá and Itaquaí Rivers, Javari Valley, State of Amazonas, Brazil. Rev Soc Bras Med Trop. 2012;45(4):45762. doi: 10.1590/s0037-86822012000400008.

8. Kourtis A, Bulterys M, Hu D, Jamieson D. HIV-HBV coinfection-a global challenge. N Engl J Med. 2012;366(19):1749-52. doi: 10.1056/ NEJMp1201796.

9. Medeot S, Nates S, Recalde A, Gallego S, Maturano E, Giordano M, et al. Prevalence of antibody to human T cell lymphotropic virus types $1 / 2$ among aboriginal groups inhabiting northern Argentina and the Amazon region of Peru. Am J Trop Med Hyg. 1999;60(4):623-629. doi: 10.4269/ ajtmh.1999.60.623.

10. Blas MM, Alva IE, Garcia PJ, Carcamo C, Montano SM, Mori N, et al. High prevalence of human T-lymphotropic virus infection in indigenous women from the Peruvian Amazon. PLoS ONE. 2013;8(9):e73978. doi 10.1371/journal.pone.0073978.

11. Cabezas-Sánchez C, Trujillo-Villarroel O, Zavaleta-Cortijo C, Culqui-Lévano D, Suarez-Jara M, Cueva-Maza N, et al. Prevalencia de la infección por el virus de hepatitis $B$ en niños menores de 5 años de comunidades indígenas de la Amazonía peruana posterior a intervenciones mediante inmunización. Rev Peru Med Exp Salud Publica. 2014;31(2):204-10.

12. Dela Hoz F, Perez L, de Neira M, Hall AJ. Eight years of hepatitis B vaccination in Colombia with a recombinant vaccine: factors influencing hepatitis B virus infection and effectiveness. Int J Infect Dis. 2008;12(2):183-189. doi: 10.1016/j.ijid.2007.06.010.

13. Blanco RY, Loureiro CL, Villalba JA, Sulbarán YF, Maes M, de Waard JH, et al. Decreasing prevalence of Hepatitis $\mathrm{B}$ and absence of Hepatitis $\mathrm{C}$ Virus infection in the Warao indigenous population of Venezuela. PLoS One. 2018;13(5):e0197662. doi: 10.1371/journal.pone.0197662.

14. Bartlett E, Zavaleta C, Fernández C, Razuri H, Vilcarromero S, Vernund S, et al. Expansion of HIV and syphilis into the Peruvian Amazon: a survey of four communities of an indigenous Amazonian ethnic group. Int J Infect Dis. 2008;12(6):e89-e94. doi: 10.1016/j.ijid.2008.03.036.

15. Garcia D, Porras A, Rico A, Alvis N, Navas MC, De La Hoz F, et al. Hepatitis B infection control in Colombian Amazon after 15 years of hepatitis $B$ vaccination. Effectiveness of birth dose and current prevalence. Vaccine. 2018;36(19):2721-2726. doi: 10.1016/j.vaccine.2017.11.004.

16. Yacoubi L, Brichler S, Mansour W, Le Gal F, Hammami W, Sadraoui A, et al. Molecular epidemiology of hepatitis B and Delta virus strains that spread in the Mediterranean North East Coast of Tunisia. J Clin Virol. 2015;72:126-32. doi: 10.1016/j.jcv.2015.10.002.

17. Ormaeche M, Whittembury A, Pun M, Suarez-Ognio L. Hepatitis B virus, syphilis, and HIV seroprevalence in pregnant women and their male partners from six indigenous populations of the Peruvian Amazon Basin, 2007-2008. Int J Infect Dis. 2012;16(10):e724-30. doi: 10.1016/j. ijid.2012.05.1032.

18. Zavaleta C, Fernández C, Konda K, Valderrama Y, Vermund S, Gotuzzo E. High prevalence of HIV and syphilis in a remote native community of the Peruvian Amazon. Am J Trop Med Hyg. 2007 Apr;76(4):703-5.

19. Matthews PC, Beloukas A, Malik A, Carlson JM, Jooste P, Ogwu A, et al. Prevalence and Characteristics of Hepatitis B Virus (HBV) Coinfection among HIV-Positive Women in South Africa and Botswana. PLoS ONE. 2015;10(7): e0134037. doi: 10.1371/journal.pone.0134037.

20. Bittencourt A, Dourado I, Filho P, Santos M, Valadão E, Alcantara L, et al. Human T-cell lymphotropic virus type 1 infection among pregnant women in northeastern Brazil. J Acquir Immune Defic Syndr. 2001;26(5):490-4. doi: 10.1097/00126334-200104150-00016.

21. Fiestas Solórzano V, Gonzáles Noriega M, Fiestas F, Cabezudo E, Suárez M, Suárez V. Evaluación de la seroprotección contra sarampión, rubéola y hepatitis B en niños menores de cinco años del Perú, 2011. Rev Peru Med Exp Salud Publica. 2012;29(4):437-43. 